\title{
La Extensión Universitaria: un espacio fundamental para el desarrollo de la gestión cultural
}

\author{
A Extensão Universitária: \\ um espaço fundamental para o desenvolvimento da gestão cultural \\ The University Extension: \\ a fundamental space for the development of cultural management
}

\author{
Ahtziri E. Molina Roldán
}

Palabras clave:

Extensión Universitaria

Gestión Cultural

Profesionalización

Resumen:

Las Instituciones de Educación Superior (IES) en el continente han delineado tres tareas sustantivas la enseñanza, la investigación y la extensión de los servicios universitarios a la población en general. Esta tercera función, fue impulsada en la Reforma Universitaria de Córdoba (1918) pues se consideró que este era el modo de regresar a las sociedades que crearon y sustentaron a las universidades cuando el acceso a este nivel educativo era muy reducido. Sin embargo, la recomposición del sistema universitario en el último siglo ha transformado los objetivos, estrategias de operación y resultados universitarios. Por un lado se propone un mayor acercamiento al mercado laboral y otro desde la nueva izquierda que replantea el compromiso social con las comunidades que tiene la educación superior. En este disímbolo panorama la extensión universitaria sigue latente como una actividad sustantiva de las IES, aun cuando los horizontes se han diversificado y en ocasiones invisibilizado. Esta ponencia tiene como cometido identificar los distintos caminos adoptados por las estructuras de extensión universitaria en México, y a partir de ello explorar las posibilidades de que la gestión cultural le brinde herramientas para su capacitación e incluso profesionalización. De este modo, ambos campos saldrían beneficiados. 


\section{Resumo:}

As Instituições de Ensino Superior (IES) no continente têm esboçado três áreas substantivas de serviços de ensino, pesquisa e extensão universitária para a população em geral. Esta terceira função foi impulsionada na Reforma Universitária de Córdoba (1918) porque se considerou que esta era a maneira de se voltar para as sociedades que criaram e sustentaram as universidades quando o acesso a este nível era muito baixo. No entanto, a reestruturação do sistema universitário, no século passado mudou os objetivos, estratégias e resultados operacionais da universidade. Por um lado se propõe estar mais perto do mercado de trabalho e, por outro, a partir da nova esquerda se reafirma o compromisso social com as comunidades que têm o ensino superior. Neste cenário, a extensão universitária segue presente como uma atividade substantiva da IES, mesmo quando os horizontes se diversificaram e, por vezes, a tornam invisível. Este artigo tem a tarefa de identificar os diferentes caminhos percorridos pelas estruturas de extensão universitária, no México, e deste para explorar as possibilidades para que a gestão cultural forneça ferramentas para sua capacitação e profissionalização. Assim, os dois campos seriam beneficiados.

\section{Palavras chave:}

Extensão universitária

Gestão cultural

Profissionalização

\section{Keywords:}

University extension

Cultural management

Professionalization

\section{Abstract:}

Higher Education Institutions on the continent have outlined three substantive tasks of teaching, research and university extension services to the general population. This third function was driven in the University Reform of Córdoba (1918) because it was felt that this was the way to return to societies that created and sustained the universities when access to this level was very reduced. However, the restructuring of the university system in the last century has changed the objectives and strategies of the universities. On the one hand closer to the labor market, and one from the new left that redefines the social commitment to the communities that have higher education. In this paradoxical scenario, university extension is still present as a substantive activity of the higher education, even when the horizons have diversified and sometimes have become invisible. This paper aims to identify the different paths taken by the structures of university extension in Mexico, and from this to explore the possibilities for cultural management will provide tools for training and even professional. Thus, both fields would benefit. 


\section{La Extensión Universitaria: un espacio fundamental para el desarrollo de la gestión cultural}

Las Instituciones de Educación Superior (IES) en América Latina promueven fundamentalmente tres tareas básicas: la docencia, la investigación y la extensión de los servicios universitarios a la población en general. La tercera función, emanó de los postulados de las Reformas de Córdoba de $1918^{i ;}$; pues se consideró que por esta vía se podía regresar a las sociedades que crearon y sustentaron a las universidades cuando el acceso a este nivel educativo era aún más reducido que en la actualidad.

Sin embargo, el sistema de educación superior en el último siglo ha tenido reestructuraciones importantes, donde el papel de las instituciones de educación superior (IES) ha pasado de ser un espacio de generación de conocimiento a un espacio de aprendizaje de competencias que le permitan al estudiante convertirse lo antes posible en una persona apta para el desarroIlo laboral. Esto sucede a las grandes tendencias internacionales que emanan del proceso de Boloniaiii donde se busca mayor internacionalización y la homologación por competencias de los estudios a nivel mundial.

La recomposición del sistema universitario en el último siglo ha transformado en gran medida los objetivos, estrategias de operación y resultados universitarios. Por un lado se propone un mayor acercamiento al mercado laboral (modelo neoliberal) y por otro lado desde la nueva izquierda que replantea el compromiso social con las comunidades que tiene la educación superior.
En este disímbolo panorama la extensión universitaria sigue latente como una actividad sustantiva de las IES, aun cuando los horizontes se han diversificado.

Este artículo tiene como cometido identificar los distintos caminos adoptados por los modelos de extensión universitaria en México, y a partir de ello explorar las posibilidades que tiene la gestión cultural para brindarle herramientas de capacitación e incluso profesionalización. Lo anterior, partiendo de que las políticas hoy en día gravitan en América Latina sobre quiénes son los actores fundamentales de la extensión universitaria. Las diferentes asociaciones nacionales de universidades se han colocado en las antípodas de hacer de cada universitario un extensionista (Argentina), o generar un servicio profesional de gestores culturales universitarios (Colombia y Brasil). La ANUIES en México no ha planteado una postura específica sobre los extensionistas y su formación, es por eso que se toma esta ocasión para plantear una oportunidad para la retroalimentación dela tercera función universitaria se retroalimente y articule con el cuerpo de conocimientos que está creando y fortaleciendo la gestión cultural.

Se entiende por extensión la relación que las instituciones de educación superior tienen con la sociedad no activa en la vida de las mismasiv (SERNA, 2004, p. 78), y es a través de este espacio que la Universidad entrega cuentas a la sociedad sobre su desempeño en las otras dos funciones. Esta área pone en contacto el conocimiento generado en las IES con las realidades sociales para el mejoramiento de la vida de las comunidades (VILLASEÑOR, 2003, p. 67).

El sello de la extensión universitaria América Latina, a diferencia de las universidades europeas y norteameri- 
canas, es que:“la extensión universitaria busca el mejoramiento de la vida, el desarrollo y el cambio social de las comunidades para alcanzar el bien común" (VALDÉS apud SERNA, 2004, p. 79). De esta forma, el perfil de la Extensión Universitaria en América Latina busca ser solidaria, democratizante e incluyente, consciente y con actitud crítica; así es como históricamente se ha caracterizado a esta función.

Aún dentro de un momento crítico en el cual los parámetros de una Educación Superior exitosa son más bien de carácter económico expresados mediante los modos de financiamiento y calidad de las IES, en el cual los principales indicadores se refieren a asuntos de docencia e investigación, insistimos en la necesidad de reconocer su importancia y hacer más eficiente la tercera función de la universidad. Pues es difícil encontrar en las tablas de evaluación de las IES que la Extensión sea considerada una parte fundamental y que se considere la valía de estas tareas para conocer la calidad de las instituciones y el impacto social de las mismas. Esta situación genera un gran demérito y falta de atención a esta tercera función sustantiva. Lo anterior se puede corroborar en informes de la educación superior, indicadores, etc., donde esta función es contemplada superficialmente. (LÓPEZ SEGRERA, 2011; Programa sectorial de Educación 2013-2018; UNESCO, 2009)

Cabe también mencionar que, parte de los rezagos existentes derivan de la escasa profesionalización de los funcionarios de la extensión universitaria. Este artículo revisa el vínculo que puede darse entre esta actividad universitaria y el cuerpo de conocimientos aportado por la gestión cultural; pues se identifican como tareas altamente afines y con la amplitud y diversidad de horizontes que plantean ambas activida- des se encuentra que son susceptibles de compartir metodologías de trabajo y también objetivos de realización.

\section{La necesidad de la tercera función}

En los últimos años, la matrícula de educación superior en todos los países se ha incrementado, haciendo posible que cada vez sea más la población que accede a los conocimientos y la cultura de las IES. En la última década, en algunos países de América Latina, la matrícula en ES se ha duplicado. En México, el crecimiento ha sido bajo en comparación con otros países de la región, como es el caso de Chile, Uruguay, Cuba y Paraguay, donde la tasa bruta de escolaridad se duplicó, mientras que en el caso nacional sólo aumentó una tercera parte de la matrícula.

Si bien, cada vez más jóvenes tienen la posibilidad de ingresar a la ES, las desigualdades acumuladas históricamente en la región son difíciles de remontar en pocos años: a principios del siglo XXI, menos de la quinta parte de la población en América Latina ha logrado acceder a la educación superior. En este sentido, la extensión universitaria tiene un rol importante que jugar en hacer llegar a más población los beneficios de la educación superior. Sin embargo, las políticas de las IES no siempre consideran a esta función como una que pueda aportar elementos para abatir el rezago .

Si contamos sólo la población que está inscrita en alguna de las IES en México, ésta únicamente representa $2 \%$ del total de la población nacional. Para resaltar aún más la problemática, la CEPAL señala que apenas el $18 \%$ de la población entre 25 y 59 años en México ha alcanzado los trece años o más de escolaridad. Ante estas cifras, es evidente que hoy en día, un gran sector de 
la población queda excluido de los conocimientos y las propuestas culturales que se socializan en las universidades e IES. Como es posible observar, hay una oportunidad importante para que la extensión haga aportaciones directas para mejorar a la sociedad, aunque frecuentemente su labor se considere más bien de carácter suntuario o superficial. Existen pocas investigaciones que reflexionen sobre el papel de la tercera función La comprensión de lo que se entiende por extensión ha variado ampliamente en los últimos años: especialmente dada la diversificación del este nivel educativo, así como la intensa participación de la iniciativa privadaiv. La particularidad de cada institución, así como los cometidos de las instituciones, han modificado las comprensiones de lo que se entiende por extensión, llevando aque, desde la década de los noventa del siglo pasado, además de las nociones tradicionales de extensión universitaria, difusión cultural, divulgación de la ciencia y enlace comunitario, se incluyera la noción de vinculación y comunicación universitaria e incluso, en ocasiones, esta ha pasado a sustituir otras de las nociones o tareas principales de la extensión en el corazón de las IES. A continuación se realizará una revisión sucinta de las nociones básicas del tema.

\section{Nociones básicas}

Para comenzar es necesario establecer una de las definiciones de uso más frecuente la de: Extensión Universitaria y/o tercera función sucede en las IES y no es susceptible de ser catalogado como docencia o investigación, ni la administración que deriva de estas otras dos funciones. Es una especie de cajón de sastre donde a partir de la noción de universalidad y apertura al conocimiento, se plantea como una posibilidad de crecimiento y proyección de las IES en muchos temas. Por lo tanto, aquí se propone que la tercera función, como la noción de cultura, responde hoy a muchos métodos de trabajo y nociones con los que dialogan las propuestas de la gestión cultural.

Como se observa, dentro de la extensión universitaria existen varios modelos y tipos de actividades que han predominado en distintas instituciones en diferentes periodos, que han impactado y definido las políticas universitarias relacionadas con esta función. Siguiendo principalmente la conceptualización de Serna, se establecen aquí los conceptos básicos de la tercera función sustantiva que este autor plantea, y además se agreganotras que se observan vigentes en la organización de las IES.

\section{Extensión universitaria}

Se entiende por extensión la relación que las instituciones de educación superior tienen con la sociedad no activa en la vida de las mismas (SERNA, Op cit, p. 78) y es a través de este espacio que:

Las universidades... deben contribuir al contacto reflexivo con la realidad, seguida del compromiso para transformarla. La extensión tiene, en potencia, una dimensión educativa de importancia excepcional... a través de la cual la Universidad debe ser una conciencia que no sólo crítica, sino que actúa [para] acompañar a las personas y grupos humanos en la búsqueda de la respuesta a sus problemas. (Idem, p. 99-100)

No obstante, al pensar en el concepto de extensión proponemos pensar en la "integración y transformación mediante la difusión, divulgación, promoción y servicios del conocimiento científico, tecnológiCo, artístico y humanístico" (ANUIES apud SERNA, 2004, p. 84) que propicie un desarrollo armónico de las sociedades. 
Por su parte Ejea y Garduño la definen como: todas aquellas actividades que no sean Docencia o Investigación. Esto abre un espectro muy amplio de lo que cabe en esta noción. Lo cual, como observaremos más adelante, implicará ciertas contradicciones entre lo que abarca una definición y la otra.

Tradicionalmente este término era el más completo, el que incluía todas las actividades; sin embargo, debido a los cambios sustantivos en las tareas de la función, esta acepción ya no es completamente incluyente. Más aún, contrario a lo que este autor plantea aquí, las definiciones de extensión universitaria en la práctica también incluyen a los propios agentes universitarios estén en activos o no. Por lo tanto, esta tarea ha dejado de ser únicamente un espacio de atención para la población externa y también se ha convertido en un modo de atender las necesidades no académicas de los universitarios; tales como son los casos de las actividades deportivas, artísticas, de salud, entre otras.

\section{Difusión cultural}

La finalidad de la difusión cultural es transmitir a la sociedad los conocimientos, resultado de las investigaciones, las concepciones de cultura nacional y universal, las costumbres, entre otras cosas, principalmente de manera unidireccional universidad-sociedad, a través de diversos instrumentos entre otros: "publicaciones, museos y exposiciones, conferencias, funciones de cine y actividades artísticas" (SERNA, Op cit, p. 87) que rara vez se realizan como respuesta a las necesidades e intereses sociales, imponiendo muchas veces visiones del mundo y modos de vida que no son compatibles con los contextos sociales e históricos. Hay que mencionar que esta tarea fue dada a las universidades previamente a la existencia de secretarías, consejos e institutos de cultura en el país. Con lo cual, la función de de "difundir la cultura" como tarea particular de las IES ahora está cuestionada (en cuanto a que tiene otras fuentes de hacerse), pues los nuevos planes de educación superior, no contemplan tal espectro en presupuestos u objetivos específicos como lo hizo en el pasado.

Como definición de trabajo se considera (retomando a GARDUÑO; EJEA, 2014) como la función dedicada a la producción y promoción de actividades artísticas, deportivas y diversas expresiones culturales, además de otras formas de conocimiento propias de los territorios donde está inserta la institución, así como la interacción con aquellas que sucedan más allá del territorio de origen.

Cabe mencionar también, que los perfiles de las actividades culturales en las universidades se han modificado principalmente en dos direcciones. Las actividades de esta función cada vez están menos dirigidas a un público general y ahora tiene dos cometidos específicos dentro del ámbito universitario. Lo cual, a su vez, responde en gran medida a los perfiles integrales por competencias que las universidades han adoptado como parte fundamental de sus planes de estudios. Siendo esta la nueva meta principal de los programas de difusión cultural, entonces se desdibuja la posibilidad de contemplar la tarea de difusión cultural como una de extensión extra muros de la cultura, sino se convierte principalmente en una opción de atención a la población universitaria.

Otra tendencia importante en el mundo de la difusión cultural es la creación de las empresas culturales universitarias (como en el caso de la Universidad de Guadalajara)vii, donde los productos culturales pasan de ser bienes y servicios públicos a formas de ingreso para ser utilizados como fuente 
de ingreso para el sostenimiento de la propia institución. Esta concepción de carácter empresarial modifica ampliamente el sector al cual está dirigida la tercera función, así como los propósitos a alcanzar; pues entre otras cosas puede desdibujar los públicos destinatarios a partir de los cuales se dirige y los objetivos que tiene al hacerlo.

\section{la ciencia}

\section{Divulgación o Comunicación de}

Según Sánchez (2003, p. 9) "la divulgación de la ciencia es una labor multidisciplinaria cuyo objetivo es comunicar... el conocimiento científico a distintos públicos voluntarios". Con ello se busca promover el análisis crítico de los hechos sociales para crear conciencia de las situaciones en las que se vive, a fin de estimular la participación política de la sociedad para que intervenga en la transformación de sus esquemas de pensamiento, exigiendo mejores condiciones de vida.

Para fines de este trabajo se le conceptualiza la divulgación o comunicación de la ciencia como la producción, promoción y comunicación del conocimiento científico de carácter básico y aplicado en forma pertinente y eficaz para el beneficio de la sociedad.

Los alcances de este modo de extensión han sido magros, pues con frecuencia los lenguajes y espacios de difusión han sido poco apropiados para hacerlo llegar a mayores sectores de la población. Sin embargo, sí se están explorando nuevos derroteros en el tema y modos de acercarse a las poblaciones. De hecho, algunas de estas propuestas son derivados de proyectos realizados con herramientas de la gestión cultural. Tales serían los casos de: "Para leer la ciencia desde México"viii, "Domingos en la ciencia"ix, así como los diferentes cursos de capacitación que se están gene- rando en diversas partes del país a nivel diplomados y maestrías.

\section{Vinculación}

La vinculación responde a la relación que se establece entre la universidad y las empresas con el fin de elevar la formación de sus estudiantes, al igual que su investigación, y así contribuir a la mejor integración a las necesidades sociales de empleo. Basándose en los conceptos de calidad y excelencia educativa, este concepto se apega a las normas del mercado para asegurar el progreso económico del entorno inmediato de la universidad. Sin embargo, muchas veces se cae en una meritocracia radicalizada y una selección cultural acotada que sirve, más bien, para la continuación de las desigualdades sociales (SERNA, Op cit, p. 97).

Aquí se lee como: Es la interacción entre la universidad y los sectores públicos, privados y sociales, mediante la prestación de servicios y productos con el fin de desarrollar la dimensión profesional de los diversos actores involucrados. También tiene esta área una marcada tendencia de incluir estudiantes en el mundo empresarial con la finalidad de que sean capaces de incorporarse al ámbito laboral lo antes posible.

Otra acepción y posible público incluido aquí es el de los egresados de la propia institución. En tiempos recientes se ha hecho mucho énfasis en saber sus destinos, con dos finalidades preponderantes: la de darle seguimiento a su trayectoria académica y, con esto, identificar la efectividad de sus programas; y por otro lado, procurar un vínculo afectivo que pueda resultar en la donación de recursos a sus alma mater y así generar otra fuente de ingreso.

La vinculación es la acepción general más reciente. La cual, si bien replantea 
el modo en que se genera la relación con la sociedad es también la definición que apunta a la empresa y a proponer que la universidad genere sus propios recursos mediante la realización de servicios al sector empresarial y también al gubernamental. Este aspecto está siendo impulsado ampliamente desde la ANUIES y la propia Secretaría de Educación Pública, ante la disminución de fondos públicos para apoyar la educación superior.

\section{Enlace comunitario}

Estas actividades tienen como público específico a los grupos sociales marginados para ofrecer una mejor calidad de vida a través de servicios de salud y de educación, asesoría técnica, entre otros servicios sociales (ANUIES apud SERNA, 2004, p. 84-85).

A partir de la división de las tareas de extensión en diversas áreas es que se acuña este campo, que inicialmente fue uno de los motivos de la generación de la extensión universitaria como función. Pues, es donde y cuando la universidad busca llegar a poblaciones que, de otro modo, no tendrían acceso al conocimiento universitario y los beneficios que este pudiera brindar a través de proyectos de alfabetización, promoción de la salud, promoción de la lectura, apoyo a productores agrícolas, proyectos de vivienda popular, etc.

Esta acepción existe en cuanto la idea de vinculación (especialmente la empresarial) se vuelve el centro de acción de la tercera función. De otro modo, casi todo puede ser entendido como enlace comunitario, entendiendo la comunidad como la sociedad que alberga y da origen a las instancias de educación superior que realizan sus tareas a nivel local.

Por lo tanto, se conceptualiza como un espacio de encuentro con las comunidades inmediatas, en donde de manera conjunta se busca atender problemáticas locales con responsabilidad social para generar un mejor desarrollo humano. Estas pueden ser las brigadas comunitarias de alfabetización, salud, mejoramiento productivo, además de servicio social, siendo respetuosos de los saberes de las comunidades.

\section{Educación continua}

Esta es un área que se caracteriza por la promoción de cursos de corto y mediano alcance para la actualización en temas de educación superior o también en la enseñanza de saberes muy solicitados (enseñanza de idiomas, capacitación en programas de cómputo, entre otras). Esta área puede estar dedicada a la enseñanza de todo tipo de población o para poblaciones específicas. Este por ejemplo, resulta un espacio ideal para mantener contacto con los egresados y mantenerlos activos en la comunidad universitaria y con ello identificados.

Esta área en muchas IES representa una buena fuente de ingreso e incluso de empleo dentro de las instituciones. Sin embargo, también se observa que debido a su naturaleza incluyente, y también de que busca hacerse de recursos, el tipo de cursos ofrecidos varía en gran medida y no siempre se responde a saberes de carácter universitarios, sino en ocasiones algunos corresponden a modas o demandas populares, aunque no siempre estén respaldados con especialistas en el tema en las casas de estudio. Lo cual, si bien puede resultar en beneficios económicos inmediatos para la institución, también puede convertirse en una fuente que aumente o disminuya el prestigio académico de la institución en cuestión, dependiendo de los cursos ofrecidos en este rubro.

\section{Comunicación universitaria}

Otra área con mucho auge recientemente es la que incluye las tareas de 
comunicación universitaria. Si bien a lo largo de la historia las Instituciones de Educación Superior han tenido licencia para medios de comunicación locales y regionales, ahora con la presencia de internet y la lógica de la web 2.0 la comunicación universitaria se ha agilizado y ha ampliado su espacio de comunicación de las tareas que realiza, así cómo ha generado espacios específicos tanto de la divulgación de las tareas universitarias, como de análisis y servicio a las comunidades donde se genera su trabajo.

Los formatos a los que responde esta área son entre los principales: radio, televisión, publicaciones periódicas, presencia en redes sociales. El universo de la comunicación universitaria es susceptible de atravesar todos los temas del conocimiento universitario y los mensajeros y destinatarios de la comunicación pueden ser todos los agentes universitarios. También, el espectro de los públicos a los cuales se dirige resulta tan abierto como la comunicación misma, y al parecer lo será aún más en futuro próximo de acuerdo a los nuevos soportes electrónicos y formas de comunicación que se generen.

\section{Atención a la comunidad universitaria}

La extensión universitaria como un espacio de vinculación de la institución con los usuarios genera una variante más, la cual es la de asegurar la promoción de tareas de típicamente de extensión universitaria como: cursos de educación continua, actividades de difusión cultural, actividades deportivas, y servicio médico para el beneficio de la comunidad universitaria, en especial los estudiantes: así como una serie de actividades más específicas como la orientación vocacional y seguimiento educativo.

Algunas universidades como la UNAM han desarrollado dependencias específicas para la atención de los universitarios, como es el caso de la Secretaría de
Atención a la Comunidad (universitaria), donde se realizan todas estas tareas desde direcciones especializadas.

El desarrollo de áreas así de específicas genera ambigüedades conceptuales de los alcances y tareas de la tercera función. Si se promueven este tipo de servicios y actividades para las personas de casa, se les considera también tareas de extensión o de formación integral. Estos dilemas son los que surgen a partir de la sectorización y especialización del trabajo de la tercera función, que se generan aún con más ahínco en esta época de especialización.

Como es posible observar la división de las tareas de la tercera función ha conducido a niveles de especialización y con ello de fragmentación entre las implicaciones que tiene está función, lo cual muy probablemente lleva a la desarticulación y la confusión de objetivos y modos de alcanzarlo, así como las poblaciones a las que está dirigido y con ello al desdibujamiento de las tareas de la tercera función.

Las nuevas dinámicas y niveles de especialización de las tareas a desarrollar en los diversos, y algunos ya muy definidos ámbitos de la tercera función universitaria, nos llevan a preguntarnos dos cosas: ¿Quiénes son los destinatarios de estas tareas? ¿Aquiénes están dirigidas? Con esta finalidad es que se revisan los postulados de los programas de la tercera función desde la UNESCO y la asociación Nacional de Universidades e Instituciones de Educación Superior.

\section{Políticas públicas de la Tercera Función}

Las definiciones trabajadas hasta ahora están en completa correlación con las políticas de la Educación Superior, tanto a niveles internacionales como 
nacionales. En ocasiones los postulados que se hacen en los distintos niveles son congruentes; en otros más están sujetos a amplias interpretaciones, y muchas otras se resuelven mediante omisión $y$, por lo tanto, falta de acción en esos sectores. A continuación se bosquejan los principios generales propuestos por UNESCO y el caso mexicano. A nivel nacional, se revisan tanto los planes estatales para el sector como la propuesta de la ANUIES (2000).

\section{UNESCO}

En la Conferencia Mundial sobre la Educación Superior de 2009 convocada por la UNESCO se considera como eje central del comuniqué final que la Educación superior debe de promover cambio social y desarrollo. Esto se ha de lograr mediante el fomento de la investigación, la innovación y la creatividad, lo cual es enfatizado en los puntos iniciales que tienen que ver con la responsabilidad social de la educación superior - en especial, la pública - y donde las tareas de la extensión son mencionadas en el segundo punto como un una responsabilidad social de las IES para resolver los desafíos mundiales sean de carácter ambiental, económico, social o cultural. Sin embargo, el tema no es mencionado en los llamamientos a la acción que se hacen al final del documento.

\section{ANUIES}

La ANUIES ha sido en México la principal instancia que ha generado reflexión y organización de las actividades de la extensión universitaria. Para 1985 generó el primer Programa Nacional de Extensión Universitaria (PNEU), en el cual logra conciliar las opiniones y necesidades del sector de los distintos asociados. Para 1990, como parte fundamental del desarrollo de la extensión generan 6 corredores culturales entre las IES cercanas entre estados, con intereses afines y objetivos comunes. Estos corredores culturales promovidos en la década de los noventa darían paso eventualmente a que la Asociación reorganizará su trabajo en las hoy establecidas regiones de la ANUIES. Es decir, el modo operativo de la ANUIES se basa en el trabajo probado que se genera desde el aparato de extensión de modo práctico y se intenta reproducir para las otras áreas. Otro elemento importante propuesta por esta instancia es el desarrollo de los modelos de extensión para cada tipo de institución, de acuerdo al perfil académico.

Además ha desarrollado la tipificación de las IES utilizada en la sección anterior, donde clasifica seis perfiles según "su naturaleza funcional académica, las áreas de conocimiento que conforman la oferta académica y los niveles de programas que conforman su oferta" (Programa Nacional de Extensión Cultural y de los Servicios, ANUIES, 2000). De este modo en el PNEU de 2000 se propone la caracterización y modos de trabajo para el sector con objetivos y estrategias más puntuales.

A pesar de que la Dirección de Fomento a la Extensión y Vinculación de la asociación ha sido la voz cantante del sector que ha logrado proponer estructuras para la organización del trabajo del sector, en noviembre de 2013 esta instancia desapareció, pues sus funciones se consideran poco relevantes y del sector. La única oficina que subsiste es la Dirección de Vinculación, comprendida como la relación con la iniciativa privada. Más aún, desapareció como forma organizativa la red nacional de Extensión Universitaria y se mantuvieron únicamente las de carácter regional, lo cual demuestra que la alineación de las políticas educativas nacionales responden al modelo neoliberal, que va en detrimento de la comprensión de la extensión universitaria como un concepto integral y 
como promotor del desarrollo social y fortalece el papel de la universidad más cercana a la empresa, pero sobre todo como productora de sus propios ingresos. Lo anterior, la pone más cerca de una instancia proveedora de servicios que de un espacio dedicado a la educación superior, al menos a partir del tipo de lazos que está generando con la comunidad en estos días.

\section{Panorama actual de la Tercera Función en México}

La generación de perfiles múltiples de las tareas de la tercera función ha derivado en diferente medida de acuerdo a las experiencias, los tamaños de la institución y los objetivos que se plantea. Entonces se observa una fragmentación de la función, y esto en términos prácticos tiende a devenir en niveles de especialización más altos implicando una mayor necesidad de profesionalización para atender el sector.

Se observa algo similar a lo que ha ocurrido en el campo cultural en nuestro país, el Estado ha dejado paulatinamente de ser el promotor principal de la actividad cultural y ha dado paso a nuevas formas de organización y espacios de interacción. Así encontramos que el Estado, ha cambiado su papel de ser el principal mecenas y proveedor a convertirse en otro agente, que si bien conserva aún sus papel hegemónico, poco a poco se está modificando y apunta cada vez más hacia un comportamiento mercantil. De los principales actores sociales que han sido agentes de este cambio son los gestores culturales. Dada la naturaleza de su trabajo y el desarrollo de nuevas estrategias y modos de trabajo es que el sector cultural se está recomponiendo y comenzando a ponderar posiciones hegemónicas que mucho tiempo se dieron por sentadas.
Así, la universidad (especialmente la pública) está teniendo que encontrar nuevos modos de financiamiento y esto eventualmente implica la redefinición de sus tareas principales, como está sucedido con la tercera función. La reorganización observada en el sector, implica también el replanteamiento de la función de la universidad (especialmente la pública) y la gestión cultural dada su propia trayectoria y necesidad de formalización y profesionalización puede aportar y de hecho ya lo hace a los nuevos caminos y desafíos planteados a la tercera función universitaria.

Uno de los trabajos iniciales planteados son los perfiles de las personas quienes están a cargo y deben de tomar decisiones, para las cuales sería apropiado tener una preparación mínima que les ayude a identificar el terreno donde están parados y las implicaciones que esto tiene. En el marco de la Reunión Nacional de Extensión Universitaria de la ANUIES noviembre del 2014 se realizó un sondeo sobre los perfiles de los funcionarios encargados de la extensión en diversas universidades del país.

Sin que esto fuera una muestra representativa, se observó que la mayoría de los entrevistados tienen una formación en el área de Humanidades y las Artes y cuentan con conocimientos (al menos básicos) en gestión cultural, es decir, la gestión cultural ya está haciendo presencia en el campo. La mayoría de las veces, estas personas encargadas han tenido una trayectoria al interior de las universidades y conocen a sus instituciones desde diferentes áreas, pues han servido varios cargos.

\section{Reflexiones finales}

La transformación de las áreas de la tercera función, y con ello el replan- 
teamiento de la función en sí misma, representa tanto un desafío institucional, como una clara muestra del sector educativo, el cual ahora está generando además de sus cometidos tradicionales de docencia e investigación y enlace comunitario, nuevas redes, ahora como órgano autogestivo que plantea relaciones pares con el sector empresarial con la venta de servicios derivados del conocimiento. En gran medida, esta nueva cara de las IES está albergada en la tercera función. Debido a lo demoledor que resultan los programas y esquemas empresariales, contar con la lógica de mercado al interior de las universidades representa disminuir o perder un espacio de libre intercambio intelectual, de construcción de conocimiento y de innovación tecnológica y social. Ante este horizonte, las lógicas de trabajo de la gestión cultural tienen muchos elementos que aportar para cuidar que el trabajo universitario se mantenga como tal.

Pues si bien, la gestión cultural tiende a impulsar los modelos autogestivos, su carácter auto-reflexivo que emana de los postulados teóricos de lo cultural la sitúa más cerca de la lógica del trabajo universitario que las lógicas y métodos empresariales. El cuidado de lo humano y de la postulación de una evaluación más cualitativa que cuantitativa, generan un ambiente de trabajo más fértil en este espacio, más cercano a los cometidos de promover la cultura como algo universal desde esta instancia de conocimiento.

Por tal motivo, se encuentra que la tercera función universitaria tiene muchas posibilidades de retroalimentarse de los postulados de la gestión cultural. De este modo, se considera que los horizontes de ambas pueden ser compartidos y capaces de fortalecerse de modo mutuo.

\section{Bibliografía:}

ASOCIACIÓN NACIONAL DE UNIVERSIDADES E INSTITUCIONES DE EDUCACIÓN SUPERIOR (ANUIES). Programa Nacional de Extensión de la Cultura y los Servicios. XXX Sesión Ordinaria de la Asamblea General de ANUIES. México: ANUIES, 2000.

CONSEJO PARA LAACREDITACIÓN DE LA EDUCACIÓN SUPERIOR A.C. México: COPAES A.C., 2010. Disponible en <http://www.copaes.org.mx/ FINAL/inicio.php>. Acceso 17 febrero 2014.

GALAZ FONTES, J.F. ; GIL ANTÓN, M. La profesión académica en México: Un oficio en proceso de reconfiguración. Revista Electrónica de Investigación Educativa, 11(2), 2009. p. 1-30.

GARDUÑO, B. ; EJEA, T. La extensión de la cultura universitaria en México: un ensayo sobre su historia, conceptualización y relevancia. In: MÉNDEZ FIERROS, H ; FELIME CUAMEA, V. (Coord). Universidad, Ciencia y Cultura: evocaciones para un saber colectivo. México: UABC, 2014. p. 51-78.

LÓPEZ SEGRERO, F. La educación superior en el mundo y en América Latina y el Caribe: principales tendencias. In: SANTOS T. dos (Coord.). América Latina y el Caribe: escenarios posibles y políticas sociales. Montevideo: FLACSO-UNESCO. 2011. p. 204-255.

MINISTERIOS EUROPEOS DE ENSEÑANZA. Espacio europeo de enseñanza superior: Declaración de Bolonia. Bolonia, 1999. Disponible en <http:// eees.umh.es/contenidos/Documentos/DeclaracionBolonia.pdf>. Acceso 12 agosto 2015.

SÁNCHEZ M., A.M. ; SÁNCHEZ M., C. Glosario de términos relacionados con la divulgación: una propuesta. In: El Muégano Divulgador, (21), p. 9. México: Dirección General de Divulgación de la Ciencia, UNAM. 2003.

SECRETARÍA DE EDUCACIÓN PÚBLICA. Programa Sectorial de Educación 2013-2018. México: Secretaría de Educación Pública. 2013.

SERNA ALCÁNTARA, G. Modelos de extensión universitaria en México. Revista de la Educación Superior, Vol. XXXIII (131), p. 77-103. 2004. Disponible en de<http://publicaciones.anuies.mx/revista/131/2/1/es/modelos-de-extension-universitariaen-mexico>. Acesso 12 diciembre 2013.

TÜNNERMAN BERNHEIM, C. El nuevo concepto de la extensión universitaria. Morelia, Michoacán: 
Universidad Michoacana de San Nicolás de Hidalgo. 2000. Disponible en <http://www.udea.edu. co/portal/page/portal/BibliotecaPortal/InformacionInstitucional/Autoevaluacion/SistemaUniversitarioExtension/NuevoConceptoExtensionUniversitaria-CarlosTunnermann.pdf>. Acesso 13 diciembre 2013.

UNESCO. Communiqué. Conferencia Mundial sobre la Educación Superior - 2009: La nueva dinámica de la educación superior y la investigación para el cambio social y el desarrollo. París, Francia: UNESCO. 2010.

UNIVERSIDAD DE GUADALAJARA. Cultura UDG. Secretaría de Vinculación y Difusión cultural, Jalisco. 2015. Disponible en <http://www.cultura.udg. $\mathrm{mx} />$. Acceso 12 de Agosto 2015.

VILLASEÑOR GARCÍA, G. La función social de la educación superior en México: la que es y la que queremos que sea. México, DF: Universidad Autónoma Metropolitana.2003. Disponible en $<$ http://csh.xoc.uam.mx/produccioneditorial/libreriavirtual/Lafuncion/Villasenor.pdf>. Acesso $18 \mathrm{di}-$ ciembre 2013.

\section{Recebido em 20/07/2015 Aprovado em 31/07/2015}

\begin{abstract}
i Ahtziri E. Molina Roldán. Doutora en Sociología. Universidad Veracruzana, México. Contato: ahtziri@gmail. com

ii Para más información ver: Reformas de Córdoba y Manifiesto Liminar. http://www.unc.edu.ar/institucional/ historia/reforma (consultado el 18/04/2015)

iii Este proceso es resultado del Convenio de Bolonia signado en 1999 por los ministerios de educación de diversos países europeos, con la finalidad de: proceso de convergencia que tenía como objetivo facilitar el intercambio de titulados y adaptar el contenido de los estudios universitarios a las demandas sociales, mejorando su calidad y competitividad a través de una mayor transparencia y un aprendizaje basado en el estudiante cuantificado a través de los créditos ECTS.
\end{abstract}

iv No se ocupan de problemas de la educación básica, a menos que se trate de alfabetización y ahora también los institutos tecnológicos se están acercando a esta tercera función. De igual manera, es importante diferenciar entre la formación universitaria de los estudiantes, cargada de humanismo y ética, a la extensión que se lleva por parte de las universidades.

v Fuente: UNESCO Institute for Statistics. http://stats. uis.unesco.org/

vi Ejea y Garduño (2014) señalan que el día de hoy en México dos terceras partes de la Educación Superior está en manos de la Iniciativa Privada, lo cual modifica drásticamente el panorama del sector y sus cometidos.

vii A partir del año 2000 se crearon las empresas culturales de la Universidad de Guadalajara, innovando con esto, el espectro de la tercera función. Para más información ver: http://www.cultura.udg.mx/culturaudg.php

viii Este es un proyecto editorial basado en concurso ensayístico promovido por varias instancias de carácter nacional, el esfuerzo es coordinado por el Fondo de Cultural Económica y la Academia Mexicana de la Ciencia. A. C.

ix Este es un programa de la Academia Mexicana de la Ciencia iniciado en 1982 en el Museo de Ciencia y Tecnología del Distrito Federal y tiene como cometido: difundir la ciencia de una manera divertida y amena entre la niñez y la juventud mexicana y con ello, coadyuvar a despertar vocaciones científicas y el interés por el cultivo y el desarrollo de la ciencia. 